\title{
Constitutive Hyperdopaminergia is Functionally Associated with Reduced Behavioral Lateralization
}

\author{
Elise Morice', Cécile Denis', Alessandro Macario', Bruno Giros*,' and Marika Nosten-Bertrand*,I \\ 'INSERM-U5 I3, Neurobiologie et Psychiatrie, Créteil Cedex, France
}

\begin{abstract}
According to the dopamine (DA) hypothesis of schizophrenia and the strong evidence for decreased cerebral lateralization in schizophrenic patients, we postulated that hyperactivity of the dopaminergic system could be associated with a reduced behavioral lateralization in mice. Mice lacking the dopamine transporter (DAT) gene were used as a genetic model of persistent hyperdopaminergia. The DAT null mutation was transferred on C57BL/6JOrl (B6) and DBA/2JOrl (D2) inbred backgrounds for more than 10 generations of backcrossing to derive three DAT strains, B6, D2, and B6 $\times \mathrm{D} 2\left(\mathrm{~F}_{1}\right)$. Adult mutant mice of the three DAT strains and their littermates were tested for paw preference using Collins' protocol. Our results demonstrated that, whatever the genetic background, persistent hyperdopaminergia directly impairs the degree of lateralization without affecting the direction. Our results support the degree of lateralization as a good candidate phenotype to further improve genetic analysis of cerebral lateralization in normal and pathological conditions.
\end{abstract}

Neuropsychopharmacology (2005) 30, 575-58I, advance online publication, 8 September 2004; doi:I 0.1 038/sj.npp. I 300570

Keywords: schizophrenia; direction and degree of asymmetry; paw preference; knockout mice; cocaine

\section{INTRODUCTION}

Anomalies in behavioral, neuroanatomical, and neurophysiological asymmetry have been widely documented in schizophrenic patients, supporting lateralization as a good candidate symptom that could contribute to clarifying schizophrenia heterogeneity (Leboyer et al, 1998). A metaanalysis of 19 studies on schizophrenia showed that the prevalence of non-right-handedness was significantly higher in patients than in healthy subjects (Sommer et al, 2001). Similarly, in the meta-analysis of three prospective followup studies, pre-schizophrenic subjects were significantly more often non-right-handed than the general population (Sommer et al, 2001). Furthermore, in vivo imaging studies of schizophrenia have provided evidence that the right-left asymmetry of the dopamine (DA) synthesis capacity and of the dopamine transporter (DAT) binding in the caudate are both lost in antipsychotic-naive patients (Hietala et al, 1999, 1995; Hsiao et al, 2003; Laakso et al, 2000). In addition, neuroleptics are able to change the balance of hemispheric activity, thus improving left-hemispheric attentional processes (for a review, see Gruzelier, 1999), and a recent study

\footnotetext{
*Correspondence: Dr M Nosten-Bertrand or Dr B Giros, INSERMU5 13, Neurobiologie et Psychiatrie, 8, rue du Général Sarrail, 94010 Créteil Cedex, France, Tel: + 33 | 498135 16, Fax: + 33। 498136 85, E-mail: nosten@im3.inserm.fr, giros@im3.inserm.fr

Received I July 2004; revised II August 2004; accepted 16 August 2004

Online publication: 18 August 2004 at http://www.acnp.org/citations/ Npp08180404307/default.pdf
}

demonstrated that haloperidol-induced downregulation of DA synthesis was significantly greater in the left than in the right striatum (Grunder et al, 2003). Finally, a highly significant correlation was reported between depressive symptoms in antipsychotic-naive schizophrenia and DA synthesis capacity in the left striatum (Hietala et al, 1999).

Taken together, these data support the hypothesis of a potential link between cerebral lateralization and schizophrenia, and suggest that abnormal asymmetry of the dopaminergic transmission could be one of the key neurobiological substrates for this functional relationship. Based on the DA hypothesis of schizophrenia, which attributes the positive schizophrenic symptoms to a hyperdopaminergic state (Carlsson and Lindqvist, 1963; Snyder, 1976), and the decreased lateralization in schizophrenic patients (Sommer et al, 2001), we hypothesized that hyperactivity of the dopaminergic system could be associated with a reduced functional brain asymmetry.

In the present study, we tested this hypothesis using knockout (KO) mice for the DAT gene (Giros et al, 1996), which are a genetic model of persistent hyperdopaminergia (Gainetdinov and Caron, 2003). Indeed, mutant DAT-KO exhibit extremely high levels of DA synthesis and turnover, with a greatly prolonged (300-fold) extracellular lifetime of DA, resulting in a five- to 10 -fold increase in basal extracellular DA levels in the striatum (Jones et al, 1998) and nucleus accumbens (Spielewoy et al, 2000a).

Several studies have provided strong evidence that alterations in DAT-mediated processes could significantly contribute to the pathogenesis of several neurological and 
psychiatric disorders; in particular, the $D A T$ has received considerable attention as a candidate gene in genetic studies of schizophrenia and attention-deficit hyperactivity disorder (Torres et al, 2003).

Like handedness in humans, paw preference in mice, as assessed in a food-reaching task, has been widely used as a robust index of functional brain asymmetry to dissect out the genetic and physiological mechanisms underlying lateralization (Cabib et al, 1995; Collins, 1975, 1985; Denenberg et al, 1991; Nielsen et al, 1997; Nosten et al, 1989; Signore et al, 1991b).

In a recent study, we reported that the genetic background of mice dramatically affects the phenotypic expression of the DAT mutation, both at the physiological and behavioral levels. For example, depending on the genetic background, it was possible to restore previously reported deficits in DAT-KO mice, such as survival, growth rate, and ability to lactate (Morice et al, 2004). In order to take the genetic background into account, behavioral lateralization was tested in three DAT strains that were obtained following backcrossing of the mutation on C57BL/6JOrl (B6) and DBA/2JOrl (D2) inbred strains (Morice et al, 2004). These progenitor strains were chosen because they are among the most genetically, physiologically, and behaviorally contrasted inbred strains (Atchley and Fitch, 1991; PuglisiAllegra and Cabib, 1997). In terms of handedness, B6 have been described as strongly lateralized, whereas D2 were found to be ambidextrous (Biddle et al, 1993; Signore et al, 1991a).

\section{MATERIALS AND METHODS}

\section{Animals and Housing Conditions}

Homozygous DAT-KO mice were obtained by homologous recombination as described previously (Giros et al, 1996). Heterozygous (HT) DAT mice were backcrossed for more than 10 generations with mice of the C57BL/6JOrl (B6) inbred strain to derive the B6-DAT congenic strain, and with mice of the DBA/2JOrl (D2) inbred strain for the D2DAT congenic strain (Orléans, La Source, France). In addition, B6-DAT-HT females were crossed with D2-DAT$\mathrm{HT}$ males to obtain the hybrid $\mathrm{B} 6 \times \mathrm{D}_{2} \mathrm{~F}_{1}$ genetic background, referred to as $\mathrm{F}_{1}$-DAT (Morice et al, 2004). For the three DAT strains, homozygous null mutants and their wild-type (WT) and HT littermates were obtained from the mating of DAT-HT mice. The genotype of the mice was determined by polymerase chain reaction (PCR) analysis as described previously (Carboni et al, 2001). Animals were weaned at 4 weeks and were then housed two to four per cage, per litter, and per gender under standard conditions, with food and water available ad libitum. Experiments were conducted during the light phase of a 12-h light/dark schedule, with lights on at $0730 \mathrm{~h}$. Within each experiment, animals were pseudo-randomized for genetic background, genotype, gender, and treatment.

For the study of cocaine-induced hyperdopaminergia, males of the C57BL/6JIco (B6) strain, 6 weeks old, were obtained from Charles River laboratories (L'Arbresle, France).

Experiments were carried out in accordance with the European Communities Council Directive (86/809/EEC) regarding the care and use of animals for experimental procedures and approved by the local ethical committee.

\section{Paw Preference Test}

Animals were tested for behavioral lateralization between 10 and 18 weeks of age, using Collins' protocol and apparatus (Collins, 1991; Signore et al, 1991b). Mice were deprived of food for $24 \pm 4 \mathrm{~h}$ and then placed individually in a transparent plastic testing chamber $(10.5 \times 6 \times 6 \mathrm{~cm})$. Small pieces of regular food were introduced in the cylindrical tube $(0.9 \mathrm{~cm}$ in diameter $)$ perpendicular to the front of the box and equally accessible using the right or left paw. Mice were observed individually for a total of 50 consecutive reaches for food. Latency of the first reach and duration to complete the test were recorded for each individual. Animals that did not start the test within a maximum of $60 \mathrm{~min}$ or that failed to complete the 50 reaches within an additional $60 \mathrm{~min}$ period were given a second trial one week later. A maximum of four trials were given per mouse. Subjects were assigned a score representing the direction of lateralization and expressed as the number of right paw entries (RPEs), with right-handed mice having an RPE score higher than 25, and left-handed mice, an RPE score lower than 25 (Collins, 1991). Scores for the degree of handedness were given by the absolute value of the difference between the number of entries with right and left paws noted $|R-L|$. Since 50 reaches for food were observed per mouse, the $|R-L|$ variable is limited to even values between 0 and 50 . Strongly lateralized (high) mice have $|R-L|$ scores between 46 and 50, whereas ambidextrous (low) individuals have $|R-L|$ scores $\leqslant 30$. Mice in the intermediate (medium) group scored between 32 and 44 . These criteria correspond to those used by Collins (1991) to select the two lines for the degree of lateralization. The initial studies in genetically uniform mice have shown that these measures of paw preferences in mice are enduring and not task specific (Betancur et al, 1991; Biddle and Eales, 1996; Collins, 1985; Nosten et al, 1989; Signore et al, 1991a,b).

\section{Effect of Cocaine on Paw Preference}

Males of the C57BL/6JIco (B6) inbred strain $(n=121)$ were split into two identical groups according to their initial scores of behavioral lateralization and were submitted to a second test of lateralization immediately after an acute injection (IP, $10 \mathrm{ml} / \mathrm{kg}$ ) of either cocaine hydrochloride $(20 \mathrm{mg} / \mathrm{kg}$, Cooper, France) or saline $(\mathrm{NaCl} 0.9 \%)$.

\section{Statistical Analyses}

As the right paw entry (RPE) and $|R-L|$ scores did not follow a normal distribution, statistical analyses were carried out using nonparametric tests (StatView software, Abacus). Multisample tests were carried out using the Kruskal-Wallis test. The Mann-Whitney rank sum test was used to compare quantitative variables between two groups. Proportions were compared using the $\chi^{2}$ test. For latency and duration of the test, data were subjected to factorial two-way ANOVA with genetic background, genotype, and treatment as between-groups factors and test as withingroup factor. Significant main effects were analyzed further 
by post hoc comparisons of means using Newman-Keuls test. Only significant statistical tests are reported in the text, with the significance established at a $P$-value $<0.05$.

\section{RESULTS}

\section{Latency and Duration of the Test}

As illustrated in Figure 1, mice of the B6-DAT strain exhibited a significant increase in the latency to initiate the paw preference test and took longer to complete the 50 reaches for food compared to the other two strains. Furthermore, both scores were dramatically increased in DAT-KO compared to their WT and HT littermates. We observed that the percentage of B6-, $\mathrm{F}_{1^{-}}$, and D2-DAT-KO that initiated the test within less than 10 min was very low $(21,65$, and $48 \%$, respectively) compared to that of the WT $(87,98$, and $93 \%)$, or the HT $(73,98$, and $98 \%)$. Similarly, while most of the WT and HT individuals of the B6-, $\mathrm{F}_{1^{-}}$, and D2-DAT strains completed the test within less than $20 \mathrm{~min}(80,98$, and $93 \%$ in the WT; 85,95 , and $85 \%$ in the $\mathrm{HT})$, the percentages were dramatically reduced in the KO mice $(24,27$, and $52 \%)$. Although the genotype-background interaction for the latency was not significant, we observed that B6-DAT-KO only took four-fold more time to initiate the test than their WT littermates, while mutants of the $\mathrm{F}_{1}$ and D2-DAT strains took five- and six-fold more time, respectively, than their respective controls.

\section{Direction and Degree of Lateralization}

As shown in Table 1, no overall effects of the genotype or of the genetic background were observed for the direction of lateralization. Whatever the genetic background and the genotype, there were as many right-handed $(\mathrm{RPE}>25)$ as left-handed subjects $(\mathrm{RPE}<25)$ in all groups of mice.

For the degree of lateralization, however (Figure 2a), both the genetic background (Kruskal-Wallis, $\mathrm{H}_{2}=13.2$, $P<0.01)$ and the genotype $\left(H_{2}=52.4, P<0.001\right)$ were significant. Partial comparisons revealed that WT of the B6- and $\mathrm{F}_{1}$-DAT did not differ and were significantly more strongly lateralized than WT of the D2-DAT strain (MannWhitney, $z=2.04, P<0.05 ; z=2.25, P<0.05$, respectively). Strikingly, for the three genetic backgrounds, DAT-KO mice

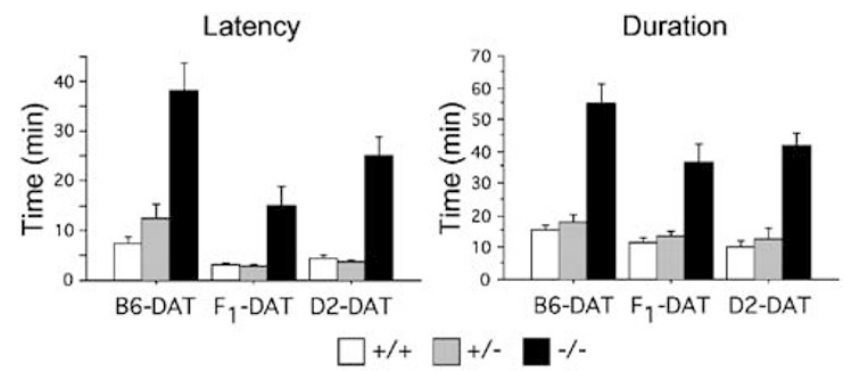

Figure I Latency and duration of the paw preference test in WT $(+/+), \mathrm{HT}(+/-)$, and $\mathrm{KO}(-/-)$ mice of the B6-, $\mathrm{F}_{1^{-}}$, and D2-DAT strains $(n=58-62$ mice per group). ANOVA for the latency: genetic background, $F_{2,529}=12.8, \quad P<0.0001$ and genotype, $F_{2,529}=49.0$, $P<0.000$ I; for the duration: genetic background, $F_{2.529}=5.5, P<0.0$ I and genotype, $F_{2,529}=72.1, P<0.0001$. Data points depict mean $\pm S E M$.
Table I Frequency of Right-Handed DAT-WT, -HT, and -KO Mice of the B6, $F_{1}$, and D2 Strains

\begin{tabular}{lcc}
\hline Background & Genotype & RPE $>\mathbf{2 5}$ \\
\hline \multirow{2}{*}{ B6-DAT } & WT & 0.47 \\
& HT & 0.42 \\
& KO & 0.62 \\
FI-DAT & WT & 0.50 \\
& HT & 0.44 \\
& KO & 0.55 \\
D2-DAT & WT & 0.57 \\
& HT & 0.53 \\
& KO & 0.53 \\
\hline
\end{tabular}

a

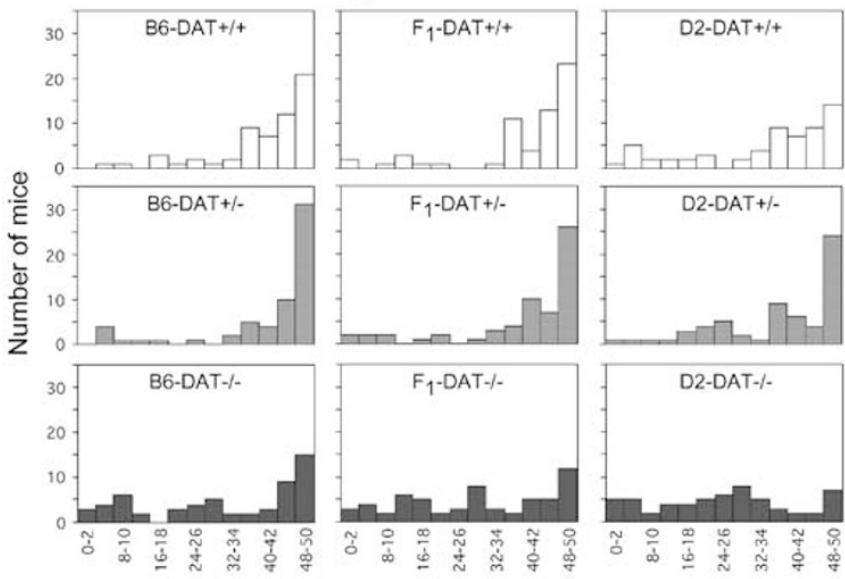

b

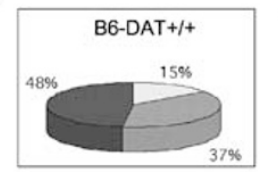

B6-DAT+1-

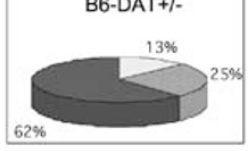

B6-DAT- $\%$
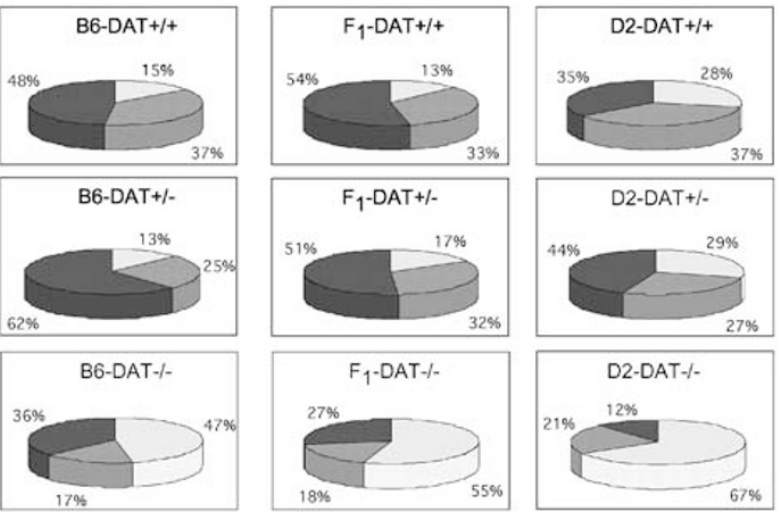

D2-DAT+1-

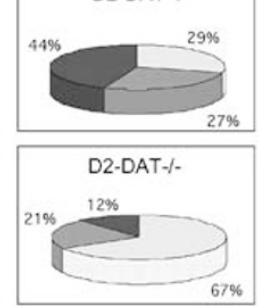

$\square \mathrm{L} \square \mathrm{M} \square \mathrm{H}$ 
were significantly less lateralized than both their WT and HT littermates. The leftward shift of the KO distributions was comparable among the three DAT strains, so that the B6-DAT-KO remained more strongly lateralized than the D2-DAT-KO (Mann-Whitney, $z=2.2, P<0.05$ ). Finally, as illustrated in Figure $2 b$, the proportions of strongly lateralized, ambidextrous, and intermediate individuals of the three $\mathrm{B} 6-, \mathrm{F}_{1^{-}}$, and D2-DAT strains differed significantly between $\mathrm{KO}$ and their WT and HT littermates.

\section{Test-Retest Experiment}

As a result of constitutively enhanced dopaminergic tone, DAT-KO mice have been described to exhibit a high novelty-driven hyperactivity (Gainetdinov et al, 1999) that could interfere with their behavior in the testing chamber box and be responsible of an initial ambidextrous behavior. In addition, DAT-KO had jerkier and less precise movements compared to the WT; this reduced skill could have modified the behavioral expression of their lateralization. In order to rule out these two possibilities, we carried out a test-retest experiment to compare the behavioral evolution among ambidextrous mice of the three genotypes. Individuals of B6-DAT strain were selected for their low ambidextrousness $(|R-L|<30)$. These animals were further submitted to three additional paw preference tests at 1-week intervals (Figure 3). We observed that the genotype and test factors as well as their interaction significantly affected both the latency and the duration of the test. DAT-KO improved their scores along successive tests and, at the fourth test, they did not differ any more from their WT and HT littermates, neither for the latency nor for the duration of the test. For the degree of lateralization, however, the test factor alone was significant (ANOVA, $\mathrm{F}_{3,176}=14.4$, $P<0.0001)$ : ambidextrousness evolution was similar among the three genotypes.

\section{Effect of Acute Cocaine Administration on Paw Preference}

Ambidextrous behavior observed in the DAT-KO adults could be the long-lasting expression of a developmentally altered brain asymmetry in response to the constitutively high dopaminergic tone. However, this ambidextrous behavior could also be functionally associated to the hyperdopaminergic tone at the very time of the test. We thus tested whether an acute cocaine-induced hyperdopaminergia could affect behavioral scores of mice in the paw preference test. Following an acute injection of cocaine (20 mg/kg), mice from the C57BL/6JIco (B6) strain exhibited a significant increase in the latency to initiate the test and took longer to complete the 50 reaches for food compared to saline-treated animals (Figure 4a). Moreover, 14 out of 60 $(23.3 \%)$ cocaine-treated animals did not complete the test. However, in terms of lateralization, cocaine treatment did not significantly modify the direction of lateralization: we observed as many right-handed mice in saline-treated as in cocaine-treated groups (0.46 vs 0.54 , respectively). In addition, despite the absence of significant effect of cocaine treatment, the distribution of the degree of lateralization in the cocaine-treated group was roughly flat compared to the J-shaped distribution in the saline group and we observed approximately twice as much low lateralized animals (L) in the cocaine group compared to the saline group (Figure $4 \mathrm{~b}$ ).

Finally, we noted that, following the saline injection, none of the strongly lateralized mice became ambidextrous and only $10 \%$ of the intermediate group became low, whereas, following the cocaine treatment, $12 \%$ of the high mice became low and 33\% intermediate became low under cocaine. It is worth noting that cocaine-treated animals that did not complete the test were equally distributed among the three classes.

\section{DISCUSSION}

The present findings clearly demonstrate that hyperactivity of the dopaminergic system is associated with a loss of behavioral lateralization, as tested in the paw preference test in DAT-KO mice. This decrease in lateralization was observed for the three DAT strains that are normally contrasted for their degree of paw preference. Indeed, WT mice of the B6-DAT strain were found to be more strongly lateralized than the D2-DAT, as previously described for the B6 and D2 inbred strains of mice (Biddle et al, 1993; Signore et al, 1991a). In addition, the DAT-F 1 hybrid strain was similar to its highly lateralized B6 parental strain, supporting a dominant model for the higher degree of lateralization. For the three strains, the DAT-HT mice did not differ from their WT littermates, indicating that their intermediate molecular dopaminergic status (Giros et al, 1996; Jones et al,
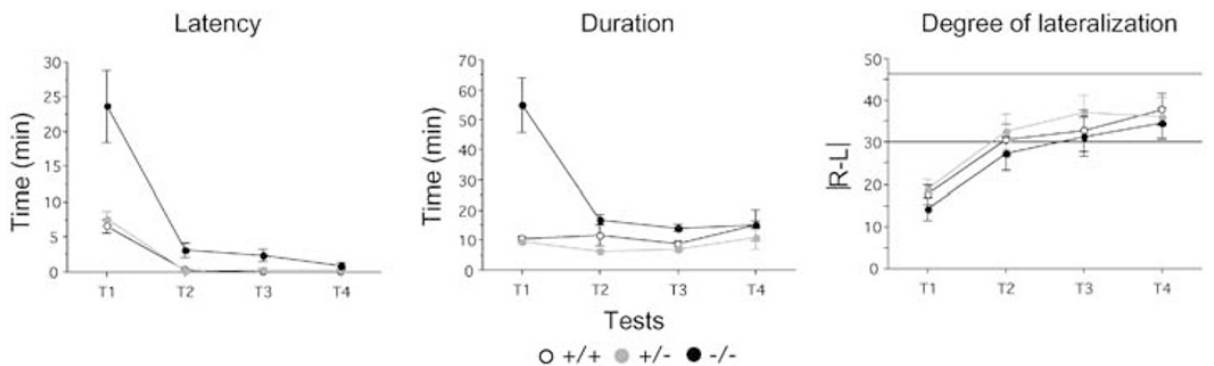

Figure 3 Latency, duration, and degree of lateralization of ambidextrous $(|R-L| \leqslant 30)$ mice as measured in a test-retest experiment with $W T$ ( $+/+$ ), $\mathrm{HT}(+/-)$, and $\mathrm{KO}(-/-)$ mice of the B6-DAT strain $(n=15-16$ mice per group). Horizontal lines in the right-handed panel indicate the criteria defining high $(|R-L| \geqslant 46)$ and low $(|R-L| \leqslant 30)$ lateralization scores. ANOVA for the latency: genotype, $F_{2,176}=16.8, P<0.0001$; test, $F_{3,176}=42.7, P<0.0001$; and interaction $F_{6,176}=7.36, P<0.000 I$; for the duration: genotype, $F_{2,176}=27 . I, P<0.000 I$; test, $F_{3,176}=\mid I .9, P<0.000 I$; and interaction $F_{6,176}=1 I .2$, $P<0.000$ I. Data points depict mean \pm SEM. 
a

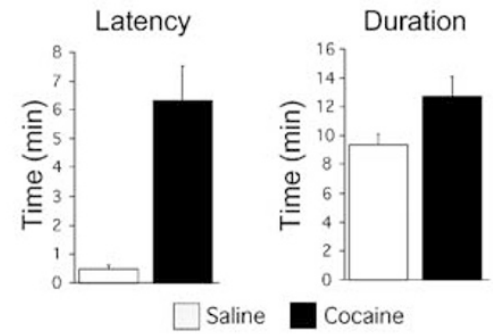

b

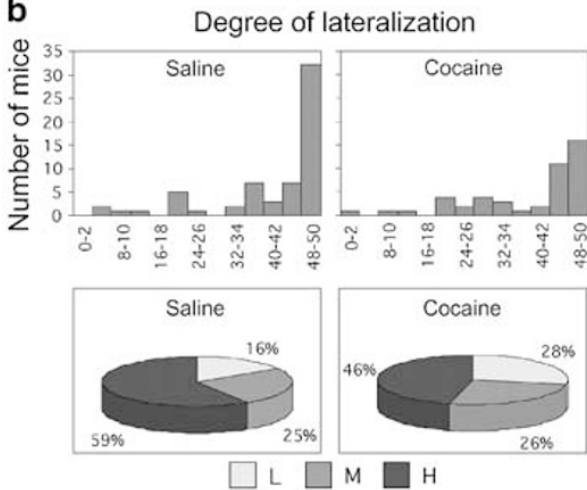

Figure 4 (a) Latency and duration of the paw preference test in C57BL/ 6)Ico (B6) mice ( $n=46-61$ mice per group) tested for behavioral lateralization following an acute injection of saline or cocaine $(20 \mathrm{mg} / \mathrm{kg})$. Student test: $t=31.8, P<0.000 \mathrm{I}$ and $t=5.87, P<0.05$, respectively. Data points depict mean \pm SEM. (b) Degree of lateralization in C57BL/6)Ico (B6) mice ( $n=46-61$ mice per group) tested following an acute injection of saline or cocaine $(20 \mathrm{mg} / \mathrm{kg})$. Mann-Whitney test: saline- vs cocaine-treated mice, $z=-1.27, P=0.204$. (Top) Distributions for the $|R-L|$ variable that takes even values from 0 to 50 with data divided into 13 classes of two values. (Bottom) Distributions for the three classes of highly $(H)$ lateralized mice $(|R-L| \geqslant 46)$, ambidextrous $(L)$ mice $(|R-L| \leqslant 30)$, and intermediate (M) mice $(32 \leqslant|R-L| \leqslant 44)$.

1999, 1998; Sora et al, 2001, 1998) was not sufficient to modify the behavioral expression of cerebral asymmetry, or that the paw preference test was not sensitive enough to detect any subtle lateralization deficit.

We have previously demonstrated that, depending on the phenotypes, the $\mathrm{B} 6, \mathrm{D} 2$, and $\mathrm{F}_{1}$ genetic backgrounds are extremely contrasted in their ability to compensate for the absence of DAT (Morice et al, 2004). For instance, whereas the mutation was extremely deleterious when expressed on the B6 genetic background, with mutants exhibiting premature death and inability to lactate, the two other backgrounds were able to compensate to such an extent that premature death was completely abolished in $\mathrm{F}_{1}$-DAT-KO mice and lactation was maintained in both D2- and $\mathrm{F}_{1}$-DATKO females. Similarly, the genetic background was able to modulate both the quantitative and qualitative expressions of the most obvious behavioral phenotype of DAT-KO mice, their spontaneous hyperactivity (Morice et al, 2004). In contrast, in the present study, we observed a similar leftward shift of the distribution of the degree of lateralization for the $\mathrm{KO}$ mice of the three DAT strains, indicating that, whatever the genetic background, the hyperdopaminergic status affects the degree of lateralization to the same extent. Consequently, the initial phenotypic difference between the two progenitor strains was maintained in the DAT-KO.
DAT-KO mice were previously shown to display a reduced rate of habituation of exploratory locomotor behavior when exposed to a novel environment (Mead et al, 2002; Spielewoy et al, 2000b). In our test-retest experiment, DAT-KO mice improved the time taken to initiate and complete the task, and they performed the task with the same efficiency as controls after four tests. Since DAT-KO mice exposed several times to the testing chamber before the test did not perform better than naive DAT-KO mice (data not shown), our results demonstrate that hyperdopaminergia does not prevent habituation when the experimental condition involves individual survival. Despite the improved attentional and motor behaviors, the population of ambidextrous $\mathrm{KO}$ mice remained ambidextrous, supporting that the ambidextrousness of DAT-KO is not dependent on their initial hyperactivity, distractibility, or reduced motor coordination. These data are in agreement with Collins' results, showing that paw preference in mice is robust and independent of the postural/motor response imposed by the test (Collins, 1985). Similarly, the slight but significant increase of the degree of lateralization in ambidextrous mice with time was expected according to Collins' (1985) analysis showing that the expression of lateralization is more labile in weakly lateralized individuals.

In addition to their obvious hyperactivity and reduced behavioral lateralization, DAT-KO mice exhibit heightened rates of stereotypies and a perseverative pattern of motor, social, and cognitive responses (Gainetdinov et al, 1999; Ralph et al, 2001; Rodriguiz et al, 2004) that are also vulnerability traits contributing to the schizophrenic syndrome (Gainetdinov et al, 2001). Moreover, DAT mutant mice also exhibit sensorimotor gating deficits as measured by prepulse inhibition (PPI) of the acoustic startle reflex (Ralph et al, 2001), which is considered a useful model for studying the neurobiology of impaired sensorimotor gating in schizophrenia, with good face, predictive, and construct validity (Swerdlow et al, 1994). Future pharmacological and genetic studies of these deficits using DAT-KO mice of different genetic backgrounds could help identify common and specific neurobiological substrates and understand the potential relationships between impaired asymmetry and cognitive deficits. In addition, the recent identification of a functional polymorphism in the tryptophan hydroxylase-2 (Tph2) gene, responsible for a $45 \%$ difference in striatal 5HTP synthesis between B6 and D2 mice (Zhang et al, 2004), illustrates the power of the genetic analysis of DAT-KO mice of different genetic backgrounds (Morice et al, 2004) to detect modifier genes that can contribute to individual differences in the susceptibility to complex disorders such as schizophrenia.

In the present experiments, cocaine, used in inbred B6 mice to induce an acute hyperdopaminergic tone at the time of the test, was shown to interfere with both the latency and the duration of the test, but had only a mild effect on the degree of behavioral lateralization. These results are in agreement with those obtained in the test-retest experiment since the two variables that are sensitive to the acute hyperdopaminergia, latency and duration, were also shown to improve with habituation. In contrast, the degree of lateralization, which is a more stable variable, appears to be more dependent on the constitutive hyperdopaminergia. 
This differential effect could also be due to the fact that these phenotypes are not affected by the same dose of cocaine. However, a dose-response study indicated that this was the highest dose at which most animals were able to complete the test (data not shown). Additional pharmacological manipulation of the DA system will thus be necessary to further identify the underlying relationship between the constitutive hyperdopaminergia and the loss of functional lateralization.

Behavioral and brain structural asymmetries can be defined by both a dimension of directionality (right/left) and a dimension of magnitude (strongly/weakly lateralized). Collins' experiments on lateralization in mice clearly established that refocusing research on the study of the degree of asymmetry significantly contributed to better understanding the extent to which genetic factors influence the expression of asymmetry (Collins, 1985). Research on lateralization in psychiatry, like in basic neurobiology, has long remained predominantly focused on the dimension of directionality, and it is only recently that a shift to the degree of lateralization has proved to be productive (Satz and Green, 1999). Ambidextrousness and mixed-handedness were found to be powerful predictors of reading disability and psychosis, and were over represented in a subgroup of schizophrenic patients with greater cognitive compromise (Cannon et al, 1997; Crow et al, 1996; David et al, 1995; Satz and Green, 1999). Recent studies in schizophrenia support a decreased cerebral asymmetry rather than altered directional asymmetry as the potential candidate phenotype of abnormal lateralization that could contribute to individual susceptibility to schizophrenia (Sommer et al, 2001). In vivo imaging studies also pointed to the loss of asymmetry as a candidate marker to better characterize schizophrenia-associated deficits (Hietala et al, 1999, 1995; Hsiao et al, 2003; Laakso et al, 2000). Our data, showing that hyperdopaminergia directly impairs the degree of lateralization without affecting the direction, further support the magnitude of lateralization as the relevant and informative variable for the study of asymmetry.

\section{CONCLUSION}

Mice were previously established as an unexcelled model for basic research aimed at understanding the genetic factors affecting lateralization. The present data, using genetically engineered DAT-KO mice, support the hypothesis that hyperactivity of the dopaminergic system is functionally associated to a loss of behavioral asymmetry. Furthermore, they support the model of DAT-KO mice as a major tool to understand the relationships among structural and functional asymmetries, and between impaired asymmetry and cognitive deficits, as well as to identify how these anomalies may contribute to specific psychopathological dimensions and clinical features.

\section{ACKNOWLEDGEMENTS}

We are grateful to C Betancur, B Etain, M Leboyer, and F Mathieu for helpful comments on the manuscript, and C Sais for her expert technical assistance. We thank L Hillard and E Rougeron for animal care. This work was supported by INSERM and the Fondation pour la Recherche Médicale. EM was supported by a fellowship from the Ministère de l'Education Nationale, de la Recherche et de la Technologie.

\section{REFERENCES}

Atchley WR, Fitch WM (1991). Gene trees and the origins of inbred strains of mice. Science 254: 554-558.

Betancur C, Neveu PJ, Le Moal M (1991). Strain and sex differences in the degree of paw preference in mice. Behav Brain Res 45: 97-101.

Biddle FG, Coffaro CM, Ziehr JE, Eales BA (1993). Genetic variation in paw preference (handedness) in the mouse. Genome 36: 935-943.

Biddle FG, Eales BA (1996). The degree of lateralization of paw usage (handedness) in the mouse is defined by three major phenotypes. Behav Genet 26: 391-406.

Cabib S, D’Amato FR, Neveu PJ, Deleplanque B, Le Moal M, Puglisi AS (1995). Paw preference and brain dopamine asymmetries. Neuroscience 64: 427-432.

Cannon M, Jones P, Murray RM, Wadsworth ME (1997). Childhood laterality and later risk of schizophrenia in the 1946 British birth cohort. Schizophr Res 26: 117-120.

Carboni E, Spielewoy C, Vacca C, Nosten-Bertrand M, Giros B, Di Chiara G (2001). Cocaine and amphetamine increase extracellular dopamine in the nucleus accumbens of mice lacking the dopamine transporter gene. J Neurosci 21: 1-4.

Carlsson A, Lindqvist M (1963). Effect of chlorpromazine or haloperidol on formation of 3methoxytyramine and normetanephrine in mouse brain. Acta Pharmacol Toxicol (Copenh) 20: 140-144.

Collins RL (1975). When left-handed mice live in right-handed worlds. Science 187: 181-184.

Collins RL (1985). On the inheritance of direction and degree of asymmetry. In: Glick SD (ed). Cerebral Lateralization in Nonhuman Species. Academic Press: London. pp 41-71.

Collins RL (1991). Reimpressed selective breeding for lateralization of handedness in mice (published erratum appears in Brain Res 1992;573(2):359). Behav Brain Res 45: 135-145.

Crow TJ, Done DJ, Sacker A (1996). Cerebral lateralization is delayed in children who later develop schizophrenia. Schizophr Res 22: 181-185.

David A, Malmberg A, Lewis G, Brandt L, Allebeck P (1995). Are there neurological and sensory risk factors for schizophrenia? Schizophr Res 14: 247-251.

Denenberg VH, Sherman GF, Schrott LM, Rosen GD, Galaburda AM (1991). Spatial learning, discrimination learning, paw preference and neocortical ectopias in two autoimmune strains of mice. Brain Res 562: 98-104.

Gainetdinov RR, Caron MG (2003). Monoamine transporters: from genes to behavior. Annu Rev Pharmacol Toxicol 43: 261-284.

Gainetdinov RR, Mohn AR, Caron MG (2001). Genetic animal models: focus on schizophrenia. Trends Neurosci 24: 527-533.

Gainetdinov RR, Wetsel WC, Jones SR, Levin ED, Jaber M, Caron MG (1999). Role of serotonin in the paradoxical calming effect of psychostimulants on hyperactivity. Science 283: 397-401.

Giros B, Jaber M, Jones SR, Wightman RM, Caron MG (1996). Hyperlocomotion and indifference to cocaine and amphetamine in mice lacking the dopamine transporter. Nature 379: 606-612.

Grunder G, Vernaleken I, Muller MJ, Davids E, Heydari N, Buchholz HG et al (2003). Subchronic haloperidol downregulates dopamine synthesis capacity in the brain of schizophrenic patients in vivo. Neuropsychopharmacology 28: 787-794.

Gruzelier JH (1999). Functional neuropsychophysiological asymmetry in schizophrenia: a review and reorientation. Schizophr Bull 25: 91-120. 
Hietala J, Syvalahti E, Vilkman H, Vuorio K, Rakkolainen V, Bergman J et al (1999). Depressive symptoms and presynaptic dopamine function in neuroleptic-naive schizophrenia. Schizophr Res 35: 41-50.

Hietala J, Syvalahti E, Vuorio K, Rakkolainen V, Bergman J, Haaparanta M et al (1995). Presynaptic dopamine function in striatum of neuroleptic-naive schizophrenic patients. Lancet 346: $1130-1131$.

Hsiao MC, Lin KJ, Liu CY, Tzen KY, Yen TC (2003). Dopamine transporter change in drug-naive schizophrenia: an imaging study with 99mTc-TRODAT-1. Schizophr Res 65: 39-46.

Jones SR, Gainetdinov RR, Hu XT, Cooper DC, Wightman RM, White FJ et al (1999). Loss of autoreceptor functions in mice lacking the dopamine transporter. Nat Neurosci 2: 649-655.

Jones SR, Gainetdinov RR, Jaber M, Giros B, Wightman RM, Caron MG (1998). Profound neuronal plasticity in response to inactivation of the dopamine transporter. Proc Natl Acad Sci USA 95: 4029-4034.

Laakso A, Vilkman H, Alakare B, Haaparanta M, Bergman J, Solin $\mathrm{O}$ et al (2000). Striatal dopamine transporter binding in neuroleptic-naive patients with schizophrenia studied with positron emission tomography. Am J Psychiatry 157: 269-271.

Leboyer M, Bellivier F, Nosten-Bertrand M, Jouvent R, Pauls D, Mallet J (1998). Psychiatric genetics: search for phenotypes. Trends Neurosci 21: 102-105.

Mead AN, Rocha BA, Donovan DM, Katz JL (2002). Intravenous cocaine induced-activity and behavioural sensitization in norepinephrine-, but not dopamine-transporter knockout mice. Eur J Neurosci 16: 514-520.

Morice E, Denis C, Giros B, Nosten-Bertrand M (2004). Phenotypic expression of the targeted null-mutation in the dopamine transporter gene varies as a function of the genetic background. Eur J Neurosci 20: 120-126.

Nielsen DM, Visker KE, Cunningham MJ, Keller RJ, Glick SD, Carlson JN (1997). Paw preference, rotation, and dopamine function in Collins HI and LO mouse strains. Physiol Behav 61: $525-535$

Nosten M, Roubertoux P, Degrelle H, Leboyer M (1989). Effect of the Tfm mutation on handedness in mice. J Endocrinol 121: R5-R7.

Puglisi-Allegra S, Cabib S (1997). Psychopharmacology of dopamine: the contribution of comparative studies in inbred strains of mice. Prog Neurobiol 51: 637-661.

Ralph RJ, Paulus MP, Fumagalli F, Caron MG, Geyer MA (2001). Prepulse inhibition deficits and perseverative motor patterns in dopamine transporter knock-out mice: differential effects of D1 and D2 receptor antagonists. J Neurosci 21: 305-313.

Rodriguiz RM, Chu R, Caron MG, Wetsel WC (2004). Aberrant responses in social interaction of dopamine transporter knockout mice. Behav Brain Res 148: 185-198.

Satz P, Green MF (1999). Atypical handedness in schizophrenia: some methodological and theoretical issues. Schizophr Bull 25: 63-78.

Signore P, Chaoui M, Nosten BM, Perez DF, Marchaland C (1991a). Handedness in mice: comparison across eleven inbred strains. Behav Genet 21: 421-429.

Signore P, Nosten-Bertrand M, Chaoui M, Roubertoux PL, Marchaland C, Perez-Diaz F (1991b). An assessment of handedness in mice. Physiol Behav 49: 701-704.

Snyder SH (1976). The dopamine hypothesis of schizophrenia: focus on the dopamine receptor. Am J Psychiatry 133: 197-202.

Sommer I, Ramsey N, Kahn R, Aleman A, Bouma A (2001). Handedness, language lateralisation and anatomical asymmetry in schizophrenia: meta-analysis. Br J Psychiatry 178: 344-351.

Sora I, Hall FS, Andrews AM, Itokawa M, Li XF, Wei HB et al (2001). Molecular mechanisms of cocaine reward: combined dopamine and serotonin transporter knockouts eliminate cocaine place preference. Proc Natl Acad Sci USA 98: 5300-5305.

Sora I, Wichems C, Takahashi N, Li XF, Zeng Z, Revay R et al (1998). Cocaine reward models: conditioned place preference can be established in dopamine- and in serotonin-transporter knockout mice. Proc Natl Acad Sci USA 95: 7699-7704.

Spielewoy C, Gonon F, Roubert C, Fauchey V, Jaber M, Caron MG et al (2000a). Increased rewarding properties of morphine in dopamine-transporter knockout mice (in process citation). Eur J Neurosci 12: 1827-1837.

Spielewoy C, Roubert C, Hamon M, Nosten-Bertrand M, Betancur C, Giros B (2000b). Behavioural disturbances associated with hyperdopaminergia in dopamine-transporter knockout mice. Behav Pharmacol 11: 279-290.

Swerdlow NR, Braff DL, Taaid N, Geyer MA (1994). Assessing the validity of an animal model of deficient sensorimotor gating in schizophrenic patients. Arch Gen Psychiatry 51: 139-154.

Torres GE, Gainetdinov RR, Caron MG (2003). Plasma membrane monoamine transporters: structure, regulation and function. Nat Rev Neurosci 4: 13-25.

Zhang X, Beaulieu JM, Sotnikova TD, Gainetdinov RR, Caron MG (2004). Tryptophan hydroxylase-2 controls brain serotonin synthesis. Science 305: 217. 